\title{
cDNA Microarray Analysis of the Differential Gene Expression in the Neuropathic Pain and Electroacupuncture Treatment Models
}

\author{
Jesang Ko*, Doe Sun Na \\ Byung Gil Hwang ${ }^{\S}$, Byung-Il Min ${ }^{\S}$ and Dong Suk Park ${ }^{\#}$ \\ Asan Institute for Life Sciences and Department of Biochemistry, University of Ulsan College of Medicine 138-736 \\ ${ }^{*}$ Department of Biochemistry and Molecular Biology, College of Medicine, Yeungnam University, Daegu 705-717 \\ ${ }^{\S}$ Department of East-West Medicine, Graduate School, Kyung Hee University, Seoul 130-701 \\ "Department of Acupuncture and Moxibustion, College of Oriental Medicine, Kyung Hee University, Seoul 130-701
}

Received 15 May 2002, Accepted 22 June 2002

Partial nerve injury is the main cause of neuropathic pain disorders in humans. Acupuncture has long been used to relieve pain. It is known to relieve pain by controlling the activities of the autonomic nervous system. Although the mechanism of neuropathic pain and analgesic effects of electroacupuncture (EA) have been studied in a rat model system, its detailed mechanism at the molecular level remains unclear. To identify genes that might serve as either markers or explain these distinct biological functions, a cDNA microarray analysis was used to compare the expression of 8,400 genes among three sample groups. Messenger RNAs that were pooled from the spinal nerves of 7 normal, 7 neuropathic pain, and 7 EA treatment rat models were compared. Sixty-eight genes were differentially expressed more than 2-fold in the neuropathic rat model when compared to the normal, and restored to the normal expression level after the EA treatment. These genes are involved in a number of biological processes, including the signal transduction, gene expression, and nociceptive pathways. Confirmation of the differential gene expression was performed by a dotblot analysis. Dot-blotting results showed that the opioid receptor sigma was among those genes. This indicates that opioid-signaling events are involved in neuropathic pain and the analgesic effects of EA. The potential application of these data include the identification and characterization of signaling pathways that are involved in the EA treatment, studies on the role of the opioid receptor in neuropathic pain, and further exploration on the role of selected identified genes in animal models.

*To whom correspondence should be addressed.

Tel: 82-2-3010-4143; Fax: 82-2-3010-4182

E-mail: jesangko@amc.seoul.kr
Keywords: Electroacupuncture, Microarray, Neuropathic pain, Opioid

\section{Introduction}

Neuropathic pain is part of the neurological disease spectrum and may be an expression of severe medical pathology (Hansson, 2002). Partial nerve injury is the main cause of neuropathic pain disorders in humans. The pain is characterized by a spontaneous burning pain that is accompanied by hyperalgesia and allodynia that lasts variable time spans (Talmoush, 1981). As already observed, this pain is often unresponsive to analgesics. Acupuncture has long been used to relieve pain. It is known to relieve pain by controlling the activities of the autonomic nervous system (Kimura and Sato, 1997). The descending inhibitory system and endogenous opioid (<biblio >) mediate this pain relief function of acupuncture (Han and Terenius, 1982). It has also been proposed that acupuncture triggers diffuse noxious inhibitory control on convergent neurons at the medullary and spinal levels (Bing et al., 1990).

Although the mechanism of neuropathic pain and analgesic effects of electroacupuncture (EA) have been studied in a rat model system, its detailed mechanism at the molecular level remains unclear. As a marker of neuronal activation of the central nervous system, the cellular fos (c-fos) expression has been widely used to monitor the change in neuronal activity that is evoked by peripheral input (Doucet et al., 1990; Ji et al. 1993; Guo et al., 1996). C-fos proto-oncogene is an immediate early-response (IER) gene, which is characterized by the rapid, stimulus - mediated induction of the expression in neuronal and non-neuronal cells (Doucet et al., 1990; Bogoyevitch et al., 2001). Besides IER genes, several opioid genes including preproenkephalin (PPE), preprodynorphine (PPD) and proopiomelanocortin (POMC) are expressed by the 
EA treatment (Lee and Beitz, 1992; Pan et al., 1994; Ji et al., 1993; Guo et al., 1996). Although evidence from recent studies indicate that several genes are involved in neuronal activities, little information is available regarding the genes that are involved in the development of neuropathic pain and the analgesic effects of EA.

In the present study, we developed a neuropathic animal model that showed causalgic signs on the tail. We analyzed the genes that were expressed differentially in the neuropathic pain model and EA treatment model using cDNA miroarray.

\section{Materials and Methods}

Experimental animals Young adult male Sprague-Dawley rats (Sam : TacN(SD)BR, 200-220 g, $\mathrm{n}=21$ ) were housed in group cages (4-5 per cage) with water and food available ad libitum. The room was light/dark (08:00 - 20:00 light, 20:00 - 08:00 dark) controlled and kept at $21-24^{\circ} \mathrm{C}$. All of the experiments were conducted in accordance with the guidelines of the International Association for the Study of Pain.

Neuropathic surgery For neuropathic surgery, the rat-tail model was used, as described previously ( $\mathrm{Na}$ et al., 1994; Kim et al., 2001). Briefly, under sodium pentobarbital anesthesia $(40 \mathrm{mg} / \mathrm{kg}$, i.p.), the right superior caudal trunk was exposed and transected at the level between the $\mathrm{S} 1$ and $\mathrm{S} 2$ spinal nerves that innervated the rat tail. To prevent the possible rejoining of the proximal and distal ends of the severed trunk, about a $2 \mathrm{~mm}$ piece of the trunk was removed from the proximal end. This surgery eliminated the S1 spinal nerve innervation to the tail via the right superior caudal trunk.

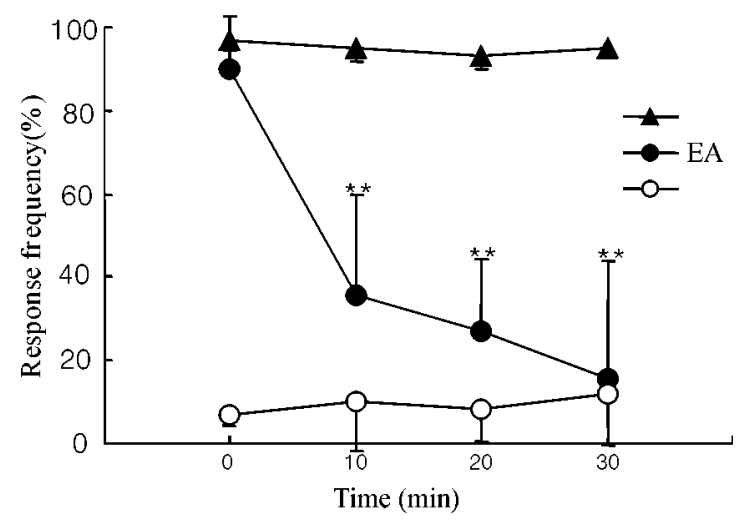

Fig. 1. Pain relief effect of EA treatment on mechanical allodynia. Three weeks after the neuropathic surgery, the control $(n=7)$ and EA treatment groups $(n=7)$ showed a high response frequency. The EA treatment group was given train-pulse $(2 \mathrm{~Hz}$, $0.3 \mathrm{~ms}$ pulse-width, $0.2-0.3 \mathrm{~mA}$ ) for $30 \mathrm{~min}$. In the EA treatment group, there were marked decreases in response frequency, whereas the control and normal sham-operation groups $(n=7)$ showed no significant changes. Asterisks indicate the values that were significantly different from the value before EA treatment ( $p<0.01$ by the Friedman rank test followed by the Dunnett posthoc test).
Behavioral test of neuropathic pain The mechanical allodynia was assessed by a normally innocuous stimulation of the tail with von Frey hair (bending force: $2.0 \mathrm{~g}$ ). For a convenient application of stimuli with von Frey hair and acupuncture, the rat was restrained in a plastic holder $(5.3 \times 15,6.3 \times 18 \mathrm{~cm}$ in diameter $\times$ length $)$, and the tail was laid on a plate. The mechanically-sensitive area was first determined by rubbing various areas of the tail with von Frey hair. The actual test was performed by gently poking the most sensitive spot with von Frey hair. An abrupt tail movement of more than $0.1 \mathrm{~cm}$ was considered an abnormal response that was attributed to mechanical allodynia. The stimulation was repeated 10 times at 10-sec intervals for each animal at each testing time or day. During the repeated trials, the test stimuli were delivered to the same spot with no difficulty, since the tail was usually stationary. The degree of response was expressed as a percentage of response frequency and was determined as follows;

Response Frequency $(\%)=\frac{\text { number of abnormal responses }}{10} \times 100$

EA stimulation Two stainless-steel needles of $0.25 \mathrm{~mm}$ diameter and $5 \mathrm{~mm}$ in length were inserted into Zusanli (ST36), which is located at the anterior tibial muscle and about $10 \mathrm{~mm}$ below the knee joint. This point produces an analgesic effect in the tail-flick response. For EA, train-pulses $(2 \mathrm{~Hz}, 0.3-\mathrm{ms}$ pulse width, 0.2-0.3 $\mathrm{mA}$ ) were applied to the inserted needle for $30 \mathrm{~min}$. The other needle (anode) was inserted into the anterior tibial muscle $5 \mathrm{~mm}$ that was distal to the first one. Anodal and cathodal leads from an electric stimulator were connected to the two acupuncture needles.

Table 1. Overall summary of the differential gene expression profile between normal and neuropathic pain models. Spinal cord mRNA was obtained from a normal rat sample, neuropathic pain model. The expression level intensity was determined using image analyzing software.

\begin{tabular}{ccc}
\hline & \multicolumn{2}{c}{ Total genes expressed differentially: } \\
& \multicolumn{2}{c}{ 11 Genes } \\
\cline { 2 - 3 } & $>2$ fold increased & $>2$ fold decreased \\
\hline Known & 5 & 5 \\
Unknown & 0 & 1 \\
Total & 5 & 6 \\
\hline
\end{tabular}

Table 2. Overall summary of the differential gene expression profile between the neuropathic pain and EA treatment models. Spinal cord mRNA was obtained from the neuropathic pain and EA treatment models. The expression level intensity was determined using image analyzing software.

\begin{tabular}{ccc}
\hline & \multicolumn{2}{c}{ Total genes expressed differentially: } \\
& \multicolumn{2}{c}{ 45 Genes } \\
\cline { 2 - 3 } & $>2$ fold increased & $>2$ fold decreased \\
\hline Known & 6 & 34 \\
Unknown & 2 & 3 \\
Total & 8 & 37 \\
\hline
\end{tabular}


Table 3. Genes decreased in the neuropathic pain model and were restored to the normal expression level after EA treatment

\begin{tabular}{|c|c|c|}
\hline & Gene Bank ID & Gene Name \\
\hline 1 & NM_011014 & opioid receptor sigma 1 \\
\hline 2 & XM_001491 & fucose-1-phosphate guanylyltransferase \\
\hline 3 & BE388188 & membrane-bound transcription factor protease, site 1 \\
\hline 4 & H99890 & TAR (HIV) RNA-binding protein 1 \\
\hline 5 & AI051561 & Homo sapiens cDNA FLJ13706 fis, clone PLACE2000317 \\
\hline 6 & AA436249 & ESTs \\
\hline 7 & AW627377 & DKFZP434N093 protein \\
\hline 8 & AK023010 & chromosome condensation 1-like \\
\hline 9 & AW241356 & hypothetical protein FLJ10261 \\
\hline 10 & AA447415 & $15 \mathrm{kDa}$ selenoprotein \\
\hline 11 & Z98044 & hexose-6-phosphate dehydrogenase (glucose 1-dehydrogenase) \\
\hline 12 & XM_013097 & zinc finger protein $6(\mathrm{CMPX} 1)$ \\
\hline 13 & BF439351 & Novel human gene mapping to chromosome 13 \\
\hline 14 & $\mathrm{AI} 312130$ & chromosome 2 open reading frame 1 \\
\hline 15 & AW503100 & UDP-glucose:glycoprotein glucosyltransferase 1 \\
\hline 16 & AI056157 & ESTs \\
\hline 17 & BF000639 & Homo sapiens cDNA: FLJ22063 fis, clone HEP10326 \\
\hline 18 & S59049 & regulator of G-protein signaling 1 \\
\hline 19 & AF196970 & Wiskott-Aldrich syndrome (eczema-thrombocytopenia) Nck associated protein \\
\hline 20 & BF055337 & chromodomain protein, Y chromosome-like \\
\hline 21 & AA494538 & ESTs \\
\hline 22 & $\mathrm{C} 06042$ & carcinoembryonic antigen-related cell adhesion molecule 7 \\
\hline 23 & AI868427 & $95 \mathrm{kDa}$ retinoblastoma protein binding protein; KIAA0661 gene product \\
\hline 24 & AW770225 & KIAA0996 protein \\
\hline 25 & NM_004461 & phenylalanine-tRNA synthetase-like \\
\hline 26 & XM_003352 & calmegin \\
\hline 27 & AW847768 & myosin phosphatase, target subunit 1 \\
\hline 28 & $\mathrm{X} 12458$ & Protein P3 \\
\hline 29 & XM_002762 & tumor necrosis factor, alpha-induced protein 6 \\
\hline 30 & BG032225 & integrin cytoplasmic domain-associated protein 1 \\
\hline 31 & BF001626 & homeo box (expressed in ES cells) 1 \\
\hline 32 & BF476140 & KIAA1018 protein \\
\hline 33 & XM_005991 & KIAA0092 gene product \\
\hline 34 & U81504 & adaptor-related protein complex 3 , beta 1 subunit \\
\hline 35 & XM_005135 & GATA-binding protein 4 \\
\hline 36 & N24973 & electron-transfer-flavoprotein, alpha polypeptide (glutaric aciduria II) \\
\hline 37 & AI816983 & ESTs, Weakly similar to unnamed protein product [H.sapiens] \\
\hline 38 & X06557 & $\mathrm{T}$ cell receptor delta locus \\
\hline 39 & AA927462 & ESTs \\
\hline 40 & AA894779 & TATA-binding protein-binding protein \\
\hline 41 & AF067420 & VPS28 protein \\
\hline 42 & AB011128 & KIAA0556 protein \\
\hline 43 & XM_013068 & diaphanous (Drosophila, homolog) 2 \\
\hline 44 & AI870850 & RAD1 (S. pombe) homolog \\
\hline 45 & AW167014 & hypothetical protein IMPACT \\
\hline 46 & BF058544 & DnaJ (Hsp40) homolog, subfamily $\mathrm{C}$, member 4 \\
\hline 47 & AA972074 & ESTs \\
\hline 48 & AI394426 & mitogen-activated protein kinase 11 \\
\hline 49 & BE745439 & sulfotransferase family, cytosolic, $1 \mathrm{~A}$, phenol-preferring, member 3 \\
\hline 50 & AA442098 & hypothetical protein dJ551D2.5 \\
\hline 51 & AI718055 & growth arrest and DNA-damage-inducible, alpha \\
\hline
\end{tabular}


Table 3. Continued

\begin{tabular}{lll}
\hline & Gene Bank ID & Gene Name \\
\hline 52 & AA507382 & ESTs \\
53 & BE856915 & Homo sapiens cDNA: FLJ23546 fis, clone LNG08361 \\
54 & BF967869 & tetraspan 3 \\
55 & AI110705 & O subunit (oligomycin sensitivity conferring protein) \\
56 & N70239 & KIAA0721 protein \\
57 & AI499593 & iroquois homeobox protein 5 \\
58 & BF515939 & GL002 protein \\
59 & AA417878 & ESTs, Weakly similar to ALU8_HUMAN ALU SUBFAMILY SX SEQUENCE \\
& CONTAMINATION WARNING ENTRY [H.sapiens] \\
60 & U12128 & protein tyrosine phosphatase, non-receptor type 13 (APO-1/CD95 \\
61 & AI572906 & (Fas)-associated phosphatase) \\
62 & AA811893 & stromal antigen 3 \\
63 & AA455079 & Homo sapiens mRNA; cDNA DKFZp761G1111 (from clone DKFZp761G1111) \\
64 & AI168277 & ESTs \\
65 & AA196322 & LIM protein (similar to rat protein kinase C-binding enigma) \\
66 & AU139950 & regulator of mitotic spindle assembly 1 \\
67 & AA905765 & ESTs \\
68 & AL136561 & Homo sapiens mRNA; cDNA DKFZp761D221 (from clone DKFZp761D221); \\
& & complete cds \\
\hline
\end{tabular}

Preparation of Tissues After 30 min of daily EA stimulation, as well as behavioral tests, the rat was sacrificed under anesthesia. The skull was then immediately opened. The brain was removed and dissected. For spinal cord isolation, a laminectomy was performed. The spinal cord at the level between S1 and S2 was carefully removed, then frozen immediately in liquid nitrogen, and kept at $-70^{\circ} \mathrm{C}$.

cDNA Microarray Analysis Gene Discovery Array Mouse version 1.1 (Incyte genomics, Palo Alto, $\mathrm{CA}$ ) was used for a cDNA microarray analysis. It consisted of one $22 \times 22 \mathrm{~cm}$ nylon filter that was spotted with 8,400 non-redundant mouse cDNA clones that was chosen from the I.M.A.G.E. collection. Total RNAs were isolated from the spinal cord using Trizol (Life Technologies, Gaithersburg, MD), followed by the isolation of mRNA using OligoTex (Qiagen, Valencia, CA). The cDNA preparation was labeled with $\left[\alpha-{ }^{32} \mathrm{P}\right] \mathrm{dCTP}$ and hybridization was performed according to the manufacturers instruction. After hybridization, the filters were exposed to X-ray film and quantified by scanning densitometry.

Dot Blot Analysis Total RNAs were isolated from the spinal cord using RNA Stat-60 (TEL-TEST, Inc., Friendswood, TX) according to the manufacturers instruction. Two $\mu \mathrm{g}$ of total RNA was denatured and dotted onto a Hybond $\mathrm{N}^{+}$nylon membrane (Amersham Pharmacia Biotech., Piscataway, NJ). Cross-linking was then performed using UV irradiation. The filters were prehybridized overnight at $42^{\circ} \mathrm{C}$ in a Northern-Max hybridization solution (Ambion, Inc., Austin, TX). Hybridization was carried out overnight at $42^{\circ} \mathrm{C}$ in the same solution that contained probes that were labeled with $\left[\alpha-{ }^{32} \mathrm{P}\right] \mathrm{dCTP}$ (DuPont NEN, $6000 \mathrm{Ci} / \mathrm{mmol}$ ) by the random primer method. The DNA probes were synthesized by a

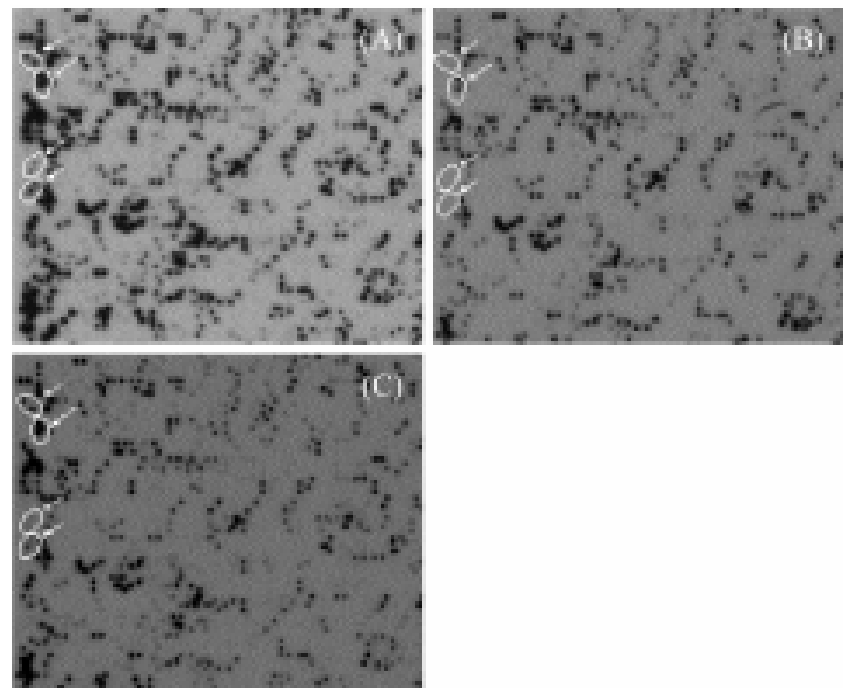

Fig. 2. Expression profile of normal (A), neuropathic pain (B), and EA treatment models (C). Spinal cord mRNA was obtained from a normal rat sample, neuropathic pain model that was developed previously (Kim et al., 2001), and EA treatment sample that was stimulated with EA for $30 \mathrm{~min}$ a day for 2 days. The expression level intensity was determined using image analyzing software. Arrows indicate examples of the differentially-expressed genes.

reverse transcriptase-polymerase chain reaction method using rat brain-derived mRNA as a template. The following probes were used in these experiments: (1) a 445-bp cDNA fragment corresponding to bases 777-1221 of the reported opioid receptor 
sigma (left primer: GCAGGACCACATCCATCTCT, right primer: TGAAGGGAAGGGCATCATAG); (2) a 401-bp cDNA fragment corresponding to bases 297-697 of the reported MAPK11 (left primer: CAGCGAAGTGTACCTCGTGA, right primer: TCAGCT GGTCGATGTAGTCG); (3) a 400-bp cDNA fragment corresponding to bases 4823-5222 of the reported APO-1/CD95 (Fas)-associated phosphatase (left primer: TTGACCCCACTTCAG TCTCC, right primer: GAGCCATATCCGGTGGTAGA); (4) a 397-bp cDNA fragment corresponding to bases 187-583 of the reported LIM protein (left primer: GACAGCACAACAGTGGCA AT, right primer: GCCCAAAGTTCTTTGCGTAG); (5) a 404-bp cDNA fragment corresponding to bases 1619-2022 of the reported Nck-associated protein (left primer: TCCACACTCACTCTGCC ATC, right primer: GGCTGTCCTGGAACTCACTC); (6) a 399-bp cDNA fragment corresponding to bases 1203-1601 of the reported iroquois homeobox protein 5 (left primer: ATCAGGAGGAGCAG AGCAGA, right primer: TTGACCTTGTCCGAGGATGT); (7) a 399-bp cDNA fragment corresponding to bases 924-1322 of the reported tumor necrosis factor $\alpha$-induced protein 6 (left primer: AGTGATGGAGATCCCTGTGC, right primer: CTCCAGTTTGA GGAGCCAAG); (8) a 402-bp cDNA fragment corresponding to bases 3098-3499 of the reported diaphanous 2 (left primer: TGTTGCATTTCTTGGCTGAG, right primer: GGTTTTCCTTGA CTGCTTGC); (9) a XbaI-HindIII-digested 500-bp fragment of glyceraldehydephosphate dehydrogenase from the pUC/GAPDH.
After hybridization, the filters were washed with $2 \times \mathrm{SSC} / 0.1 \%$ SDS for $20 \mathrm{~min}$ at room temperature, $2 \times \mathrm{SSC} / 0.1 \% \mathrm{SDS}$ at $42^{\circ} \mathrm{C}$ for $30 \mathrm{~min}$, and $0.5 \times \mathrm{SSC} / 0.1 \% \mathrm{SDS}$ for $30 \mathrm{~min}$ at $52^{\circ} \mathrm{C}$.

Data analysis Data were represented as means \pm standard error. The significance of the statistical differences was determined using the Friedman rank test, followed by the Dunnett post-hoc test in a group. $p<0.05$ was considered significant.

\section{Results and Discussion}

Pain relief effect of EA on mechanical allodynia Recently, several behavioral models for peripheral neuropathy that were produced in a rats tail were developed. We used a previously-developed rat model for neuropathic pain ( $\mathrm{Na}$ et al., 1994; Kim et al., 2001). The sign of mechanical allodynia appeared 1 day after surgery; maximal allodynia was observed in 2-3 weeks (Hwang et al., 2002). Following this result, EA was applied three weeks after surgery. Behavioral tests of mechanical allodynia were performed before and during EA at 10-min interval. In the EA-treatment group $(n=7)$, there were marked decreases in the response frequency (90.014.1, 35.724.4, 27.118.0 and 15.728.8\%, respectively). The Dunnett post-hoc test, after the Friedman rank test, indicated that there
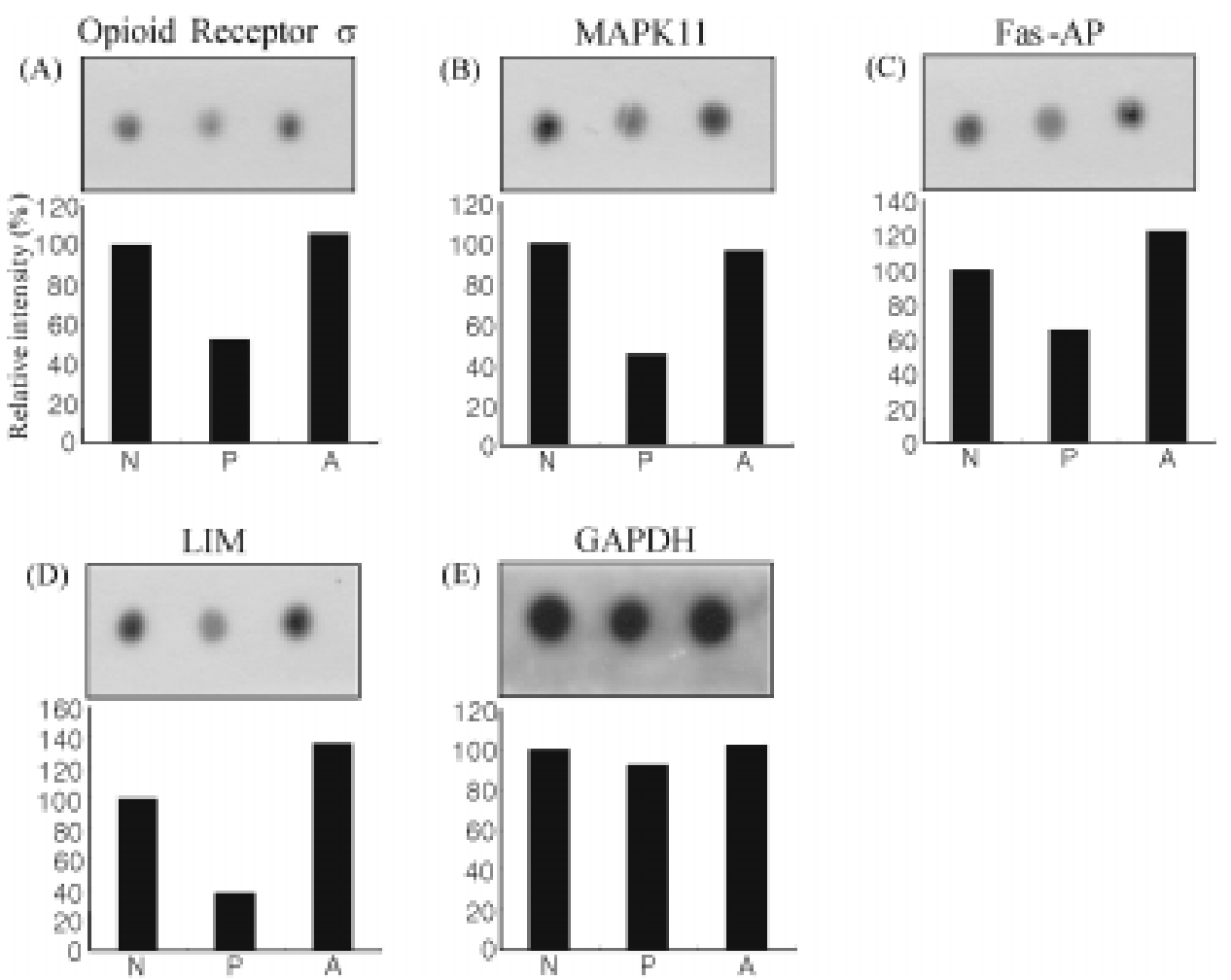

Fig. 3. Genes involved in signal transduction are expressed differentially in the pain model and restored after EA treatment. Total RNAs $(2 \mu \mathrm{g})$ were isolated from the spinal cord that was obtained from the normal $(\mathrm{N})$, neuropathic pain $(\mathrm{P})$, and EA treatment models $(\mathrm{A})$. After the total RNAs were dotted on nylon filters, the blots were hybridized with ${ }^{32} \mathrm{P}$-labeled probes. The probes that were used in the dot-blot analysis were opioid receptor sigma (A), MAP kinase 11 (B), APO-1/CD95 (Fas)-associated phosphatase (C), and LIM protein (D). GAPDH (E) total RNAs were used as a control. 
were statistically significant differences at 10,20 , and $30 \mathrm{~min}$ from the initiation of EA. The maximal pain relief effect of EA on mechanical allodynia was shown at $30 \mathrm{~min}$ after the initiation of EA (Fig. 1).

cDNA microarray analysis To identify genes that might either serve as markers or explain neuropathic pain development, or the analgesic effects of EA, a cDNA microarray analysis was used to compare the expression of 8,400 genes among three sample groups. Messenger RNAs were compared that were pooled from the spinal nerves of 7 normal, 7 neuropathic pain, and 7 EA treatment rat models. Eleven genes were identified as differentially-expressed genes between the normal and neuropathic pain models (Table 1). Among these 11 genes, 5 genes increased and 6 genes decreased in the pain model. These genes were involved in the transcription, protein synthesis, and degradation process (data not shown). Table 2 shows that the gene numbers were expressed differentially between the neuropathic pain and EA treatment models. Among 45 genes, 8 genes increased and 37 genes decreased in the EA treatment rat model, compared to the neuropathic pain rat model. These genes include receptors, signaling molecules, protein kinases, and chaperonic proteins (data not shown).

Since acupuncture treatment is known to relieve neuropathic pain, we focused on the genes that were differentially expressed in the pain model when compared to the normal, and restored to the normal expression level after the EA treatment. As shown in Table 3, 68 genes were differentially expressed more than 2-fold in the neuropathic rat model when compared to the normal, and restored to the normal expression level after the EA treatment. These genes are involved in a number of biological processes, including signal transduction, gene expression, and nociceptive pathway. The genes that were involved in the signal transduction pathway include the mitogen-activated protein (MAP) kinase (Kim et $a l$.), protein tyrosine phosphatase, protein kinase c binding protein, regulator protein of the G-protein signaling, and $\mathrm{T}$ cell receptor. The genes that were involved in the gene expression regulation include the zinc finger protein 6 and TATA-binding protein-binding protein. Interestingly, the opioid receptor sigma was one of those genes that was expressed differentially in the neuropathic pain model, and restored to the normal level after the EA treatment (Table 3). However, one third of the genes that were identified were unknown genes, or functionally uncharacterized genes.
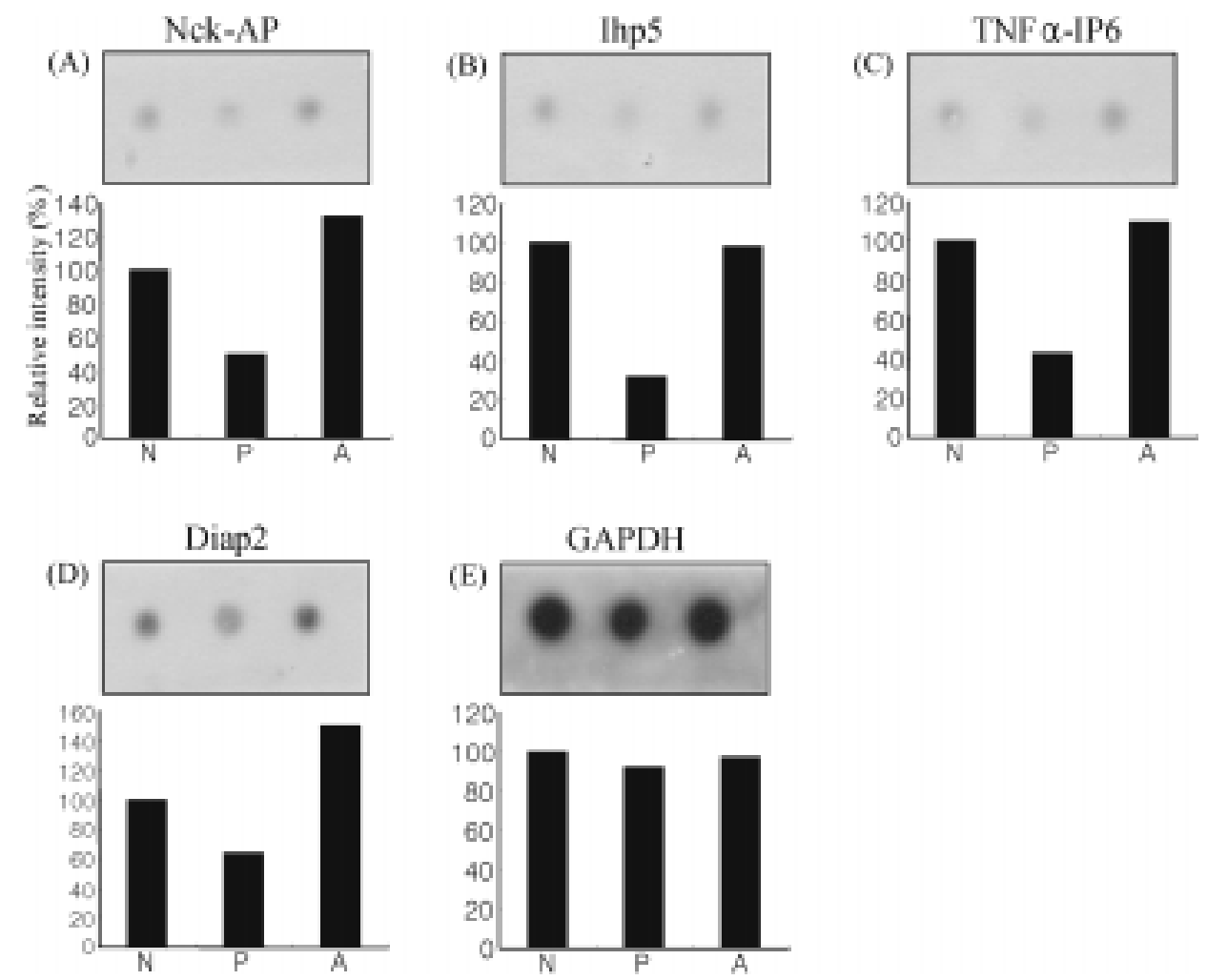

Fig. 4. The genes that were involved in the various biological processes are expressed differentially in the pain model and restored after EA treatment. Total RNAs $(2 \mu \mathrm{g})$ were isolated from the spinal cord that was obtained from normal $(\mathrm{N})$, neuropathic pain (P), and EA treatment models (A). After the total RNAs were dotted on nylon filters, the blots were hybridized with ${ }^{32} \mathrm{P}$-labeled probes. The probes that were used in the dot-blot analysis were Nck-associated protein (A), iroquois homeobox protein 5 (B), tumor necrosis factor $\alpha$ induced protein $6(\mathrm{C})$, and diaphanous 2 (D). GAPDH (E) total RNAs were used as a control. 
Opioid receptor is involved in analgesic process of EA Confirmation of the differential gene expression was performed by a dot-blot analysis. Among 68 genes that we considered, the mRNA expression level of 8 genes was downregulated in the neuropathic pain model when compared to the normal rat, and the mRNA expression level of these genes was restored to the normal state in the EA treatment sample. Results from dot blotting showed that the opioid receptor sigma was one of those genes, indicating that the opioid signaling event is involved in neuropathic pain and the analgesic effects of EA. The expression level of the opioid receptor sigma gene decreased about $50 \%$ in the neuropathic pain model. However, 1 day after the EA treatment, the mRNA expression level of the opioid receptor sigma was restored to its normal expression level. This result indicates that the cells in the spinal cord cannot normally transduce the signal in response to opioid stimulation since the opioid receptor is down-regulated in the neuropathic pain model. However, the EA treatment restores the expression level of the opioid receptor in response to the opioid signal that gives rise to analgesic effects. It was reported that endogenous opioids may be implicated in acupuncture analgesia (He, 1987). For example, acupuncture produces an increase in the opioid-like immunoreactivity in the CSF of patients with chronic pain from different sources (Ho and Wen, 1989). Our data suggest that the opioid receptor probably plays an important role in the development of neuropathic pain and the analgesic effects of EA.

Besides the opioid receptor, several interesting genes, such as the MAP kinase, zinc finger protein, and tyrosine phosphatase, were identified. The expression level of these genes decreased about 40-60\% in the neuropathic pain model. However, 1 day after the EA treatment, the mRNA expression level of these genes was restored to the normal expression level. These genes are known to function on important signal transduction pathways and gene expressions. Therefore, multiple signaling pathways, including opioid receptor- and MAP kinase-mediated pathways, as well as other gene expressions, might be involved in the pain development and analgesic effects of EA.

While further studies are required in order to delineate the signaling pathways that lead to chronic neuropathic pain and pain relief by acupuncture, this is the first report that analyzes the genes that are expressed differentially in the neuropathic pain and EA treatment models. From this information, we can begin to understand the molecular mechanism of pain development and analgesic effects of acupuncture, which are important events in pathological processes. The potential application of these data include the identification and characterization of signaling pathways that are involved in acupuncture treatment, studies on the role of the opioid receptor in neuropathic pain, and further exploration on the role of selected identified genes in animal models.

Acknowledgments This study was supported by a grant from the Oriental Medicine R\&D Project, Ministry of Health $\&$ Welfare, Republic of Korea. (HMP-00-CO-01-0001)

\section{References}

Basbaum, A. I. and Fields, H. L. (1984) Endogenous pain control systems. Annu. Rev. Neurosci. 7, 309-338.

Bing, Z., Villenueva, L. and Le Bars, D. (1990) Acupuncture and diffuse noxious inhibitory controls: naloxone-reversible depression of activities of trigeminal convergent neurons. Neurosci. 37, 809-818.

Bogoyevitch, M. A., Thien, M. and Ng, D. C. H. (2001) Characterization of protein kinases activated during treatment of cells with okadaic acid. J. Biochem. Mol. Biol. 34, 517-525.

Doucet, S. P., Squinto, S. P. and Bazan, N. G. (1990) Fos-Jun and the primary genomic response in the nervous system: possible physiological role and pathophysiological significance; in Molecular Neurobiology, Bazan, N. G. (ed.), pp. 27-55, Human, Clifton, New Jersey.

Guo, H. F., Cui, X., Hou, Y., Tian, J., Wang, X. and Han, J. (1996) C-Fos proteins are not involved in the activation of preproenkephalin gene expression in rat brain by peripheral electric stimulation (electroacupuncture). Neurosci. Lett. 207, 163-166.

Han, J. S. and Terenius, L. (1982) Neurochemical basis of acupuncture analgesia. Annu. Rev. Pharmacol. Toxicol. 22, 193220.

Hansson, P. (2002) Neuropathic pain: clinical characteristics and diagnostic workup. Eur. J. Pain 6, 47-50.

He, L. F. (1987) Involvement of endogenous opioid peptides in acupuncture analgesia. Pain 31, 99-121.

Ho, W. K. and Wen, H. L. (1989) Opioid-like activity in the cerebrospinal fluid of pain patients treated by electroacupuncture. Neuropharmacol. 28, 961-966.

Hwang, B. G., Min, B. I., Kim, J. H., Na, H. S. and Park, D. S. (2002) Effects of electroacupuncture on the mechanical allodynia in the rat model of neuropathic pain. Neurosci. Lett. 320, 49-52.

Ji, R. R., Zhang, Z. W., Zhou, Y., Zhang, Q. and Han, J. S. (1993) Induction of c-fos expression in the rostral medulla of rats following electoacupuncture stimulation. Int. J. Neurosci. 72, 183-191.

Kim, H.-H., Kim, H.-M., Kwack, K. B., Kim, S. W. and Lee, Z. H. (2001) Osteoclast differentiation factor engages the PI 3kinase, p38, and ERK pathways for avian osteoclast differentiation. J. Biochem. Mol. Biol. 34, 421-427.

Kim, H. J., Na, H. S., Back, S. K. and Hong, S. K. (2001) Sympathetic sprouting in sensory ganglia depends on the number of injured neurons. Neuroreport 12, 3529-3532.

Kimura, A. and Sato, A. (1997) Somatic regulation of autonomic functions in anesthetized animals-neural mechanisms of physical therapy including acupuncture. Jpn. J. Vet. Res. 45, 137-145.

Lee, J. -H. and Beitz, A. J. (1992) Electroacupuncture modifies the expression of c-fos in the spinal cord induced by noxious stimulation. Brain Res. 577, 80-91.

Na, H. S., Han, J. S., Ko, K. H. and Hong, S. K. (1994) A behavioral model for peripheral neuropathy produced in a rat's tail by inferior caudal trunk injury. Neurosci. Lett. 177, 50-52. 
Pan, B., Castro-Lopes, J. M. and Coimbra, A. (1994) C-fos expression in the hypothalamo-pituitary systems induced by electroacupuncture or noxious stimulation. Neuroreport 5,
1649-1652.

Talmoush, A. J. (1981) Causalgia: redefinition as a clinical pain syndrome. Pain 10, 187-197. 